\title{
Graphene Micro-roll Aptasensor for Three-Dimensional Protein Detection
}

\author{
Yuko Ueno $^{1}$, Tetsuhiko Teshima ${ }^{1}$, Calum Henderson ${ }^{1,2}$, and Hiroshi Nakashima ${ }^{1}$ \\ ${ }^{1}$ NTT Basic Research Laboratories, NTT Corporation, \\ 3-1 Morinosato Wakamiya, Atsugi, Kanagawa 243-0198, JAPAN \\ ${ }^{2}$ School of Chemistry, University of Edinburgh, \\ David Brewster Road, Edinburgh, EH9 3FJ, UK \\ ueno.yuko@lab.ntt.co.jp
}

\begin{abstract}
:
Protein detection is demonstrated by using a fluorescence graphene aptasensor formed on the inner wall of a micro cylinder (micro-roll). The graphene aptasensor is incorporated with self-foldable flexible films, leading to a three-dimensional (3D) sensing system. Since the formation of the micro-roll avoids any cytotoxic processes, the micro-roll can be used as a container to encapsulate living cells. The performance of the micro-roll graphene aptasensor is successfully confirmed while multiple cells are simultaneously encapsulated.
\end{abstract}

Key words: graphene, aptamer, protein detection, biosensor, fluorescence

\section{Introduction}

We have developed a biosensor that works on a graphene surface for the detection of biologically important proteins such as cancer markers (Fig. 1(a)) [1-2]. In our system, the graphene surface is modified with a pyreneaptamer-dye probe. These three components of the probe work as a linker to the graphene surface, a protein recognition part, and a fluorescence detection tag, respectively. Here, graphene behaves simultaneously as both an efficient acceptor for fluorescence resonance energy transfer (FRET) and as a strong adsorbate for aptamers. The graphene aptasensor detection mechanism is as follows. In the initial stage, the dye-conjugated aptamer is adsorbed on the graphene surface via $\pi-\pi$ interactions, and thus the dye is located close to the graphene surface. Here, the fluorescence of the dye is well quenched by the graphene and is barely visible (Fig. 1(a, left)). If the target of the aptamer is present in the system, the aptamer forms a complex with the target and leaves the graphene surface. At the same time, the dye molecule also leaves the graphene surface and the dye recovers its fluorescence (Fig. 1(a, right)). The target can be detected within a minute simply by adding a sample smaller than $1 \mu \mathrm{L}$ to a sensor chip. The system allows us to perform real-time molecular detection on a solid surface, which is (a)

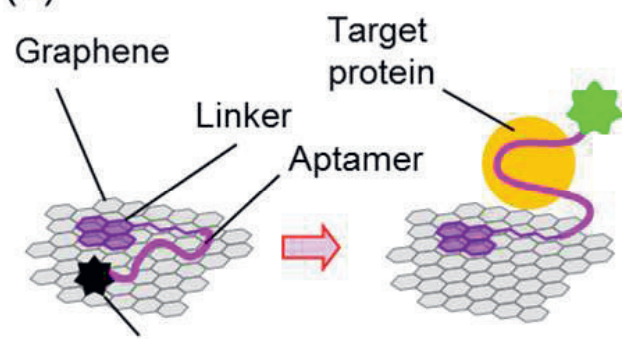

Fluorescence dye

(b)

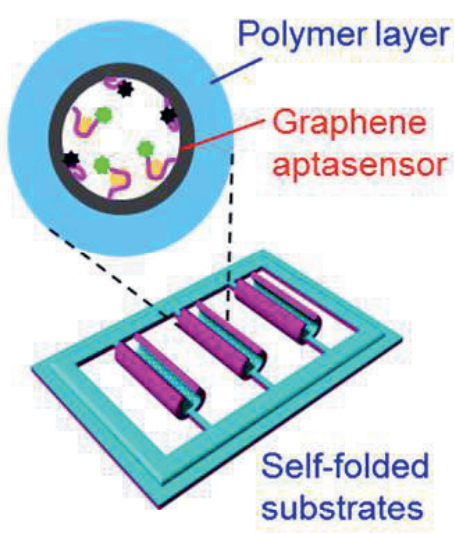

Fig. 1. (a) Design of protein detection system on graphene aptasensor, (b) schematic image of $3 D$ protein detection in a micro-roll graphene aptasensor 
advantageous for realizing a two-dimensional (2D) on-chip sensor. However, if we want to specify when and where the protein was generated by three-dimensional (3D) objects such as a cell, we need a 3D sensing platform that covers the objects.

In this paper, we extend the biosensing platform from a $2 \mathrm{D}$ plane to a hollow $3 \mathrm{D}$ space (microroll) by building the protein detection system on the inner surfaces of flexible layered polymer films (Fig. 1(b)). The different strain gradients of the polymeric bilayer are used as the driving force behind the 3D transformation to microrolls [3]. Since the formation of the micro-roll avoids any cytotoxic processes, they can be used for encapsulating cells in vitro [3].

\section{Experimental}

The micro-roll graphene aptasensor was fabricated as follows. A glass cover slip (substrate) was coated with a Ca-alginate layer, and then with a silk-fibroin hydrogel. A parylene-C layer was then deposited on it. Finally, a graphene monolayer was transferred onto the top of the trilaminar layers. To form the micro-roll, rectangular micro-patterns were fabricated via photolithography. Then, the graphene surface was functionalised with a linker and an aptamer to act as the aptasensor. In this work, we used a thrombin aptamer to prepare the aptasensor. The Ca-alginate sacrificial layer was disollved by adding ethylene-diaminetetraacetic acid (EDTA). The released polymer layers bent to form a microroll with the graphene layer inside. To encapsulate cells in a micro-roll, we placed the cells onto the functionalised graphene surface prior to adding EDTA. In this work, we used primary rat cardiomyocytes cells.

\section{Results and Discussion}

We confirmed that multiple cells were successfully encapsulated inside the micro-roll graphene aptasensor with a diameter of about $40 \mu \mathrm{m}$ (Fig 2(a)). Then, we used the micro-roll graphene aptasensor to measure the change in the fluorescence intensity that occured after adding thrombin solution into the system (Fig. 2(b), (c)). A certain level of the background fluorescence intensity was observed before adding the thrombin due to some scattering by the cell culture liquid, but after adding thrombin, the fluorescence intensity increased significantly inside the micro-roll (Fig. 2(d)). Thus, protein detection in a micro-roll graphene aptasensor has been successfully demonstrated. We can expect that in the near future it will be possible to detect proteins generated by the cells encapsulated in the graphene micro-roll aptasensor.
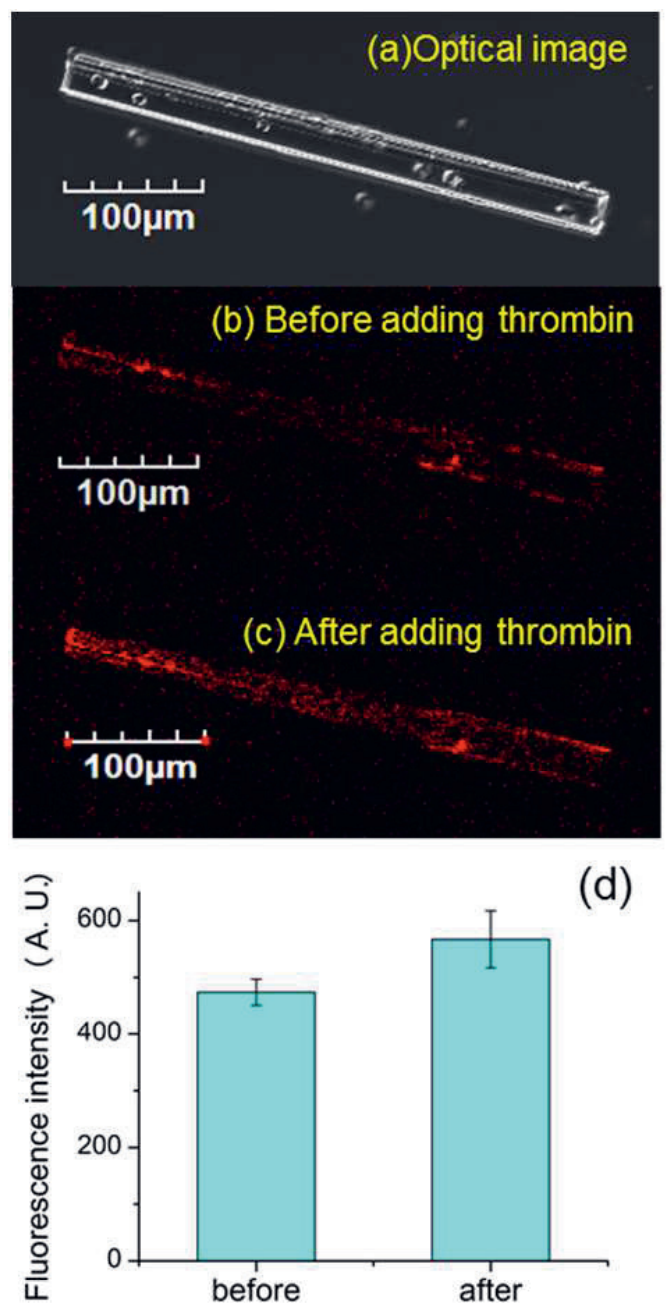

Fig. 2. Optical image of a micro-roll graphene aptasensor encapsulating primary rat cardiomyocytes, (b) fluorescence images obtained before and (c) after adding thrombin, and (d) average fluorescence intensity of several micro-roll graphene aptasensors. The error bars correspond to the standard deviations.

\section{References}

[1] Y. Ueno, K. Furukawa, K. Matsuo, S. Inoue, K. Hayashi, H. Hibino, On-chip graphene oxide aptasensor for multiple protein detection, Anal. Chim. Acta, 866, 1-9 (2015); doi: 10.1016/j.aca.2014.10.047.

[2] K. Furukawa, Y. Ueno, M. Takamura, H. Hibino, Graphene FRET Aptasensor, ACS Sens. 1, 710716 (2016); doi: 10.1021/acssensors.6b00191.

[3] T. F. Teshima, H. Nakashima, Y. Ueno, S. Sasaki, C. S. Henderson, S. Tsukada, Cell Assembly in Self-foldable Multilayered Soft Micro-rolls, Sci. Rep., 7: 17376 (2017); doi:10.1038/s41598-01717403-0.

\section{Ackowledgements}

This work was supported by JSPS KAKENHI Grant Number JP17H02759. 